\title{
Color dependence of tensor and scalar glueball masses in Yang-Mills theories
}

\author{
Ed Bennett๑, ${ }^{1}$ Jack Holligan $\odot,{ }^{2,3}$ Deog Ki Hong $\odot,{ }^{4, *}$ Jong-Wan Lee, ${ }^{4,5}$ C.-J. David Lin $\odot,{ }^{6,7}$ \\ Biagio Lucini $\odot,{ }^{8,1}$ Maurizio Piai $\odot,{ }^{2}$ and Davide Vadacchino $\odot^{9}$ \\ ${ }^{1}$ Swansea Academy of Advanced Computing, Swansea University, \\ Bay Campus, SAl 8EN Swansea, Wales, United Kingdom \\ ${ }^{2}$ Department of Physics, College of Science, Swansea University, \\ Singleton Park, SA2 8PP Swansea, Wales, United Kingdom \\ ${ }^{3}$ The Institute for Computational Cosmology (ICC), Department of Physics, \\ South Road, Durham DH1 3LE, United Kingdom \\ ${ }^{4}$ Department of Physics, Pusan National University, Busan 46241, Korea \\ ${ }^{5}$ Extreme Physics Institute, Pusan National University, Busan 46241, Korea \\ ${ }^{6}$ Institute of Physics, National Chiao-Tung University, 1001 Ta-Hsueh Road, Hsinchu 30010, Taiwan \\ ${ }^{7}$ Centre for High Energy Physics, Chung-Yuan Christian University, Chung-Li 32023, Taiwan \\ ${ }^{8}$ Department of Mathematics, College of Science, Swansea University, \\ Bay Campus, SA1 8EN Swansea, Wales, United Kingdom \\ ${ }^{9}$ INFN, Sezione di Pisa, Largo Pontecorvo 3, 56127 Pisa, Italy
}

(Received 1 May 2020; accepted 8 July 2020; published 20 July 2020)

\begin{abstract}
We report the masses of the lightest spin- 0 and spin-2 glueballs obtained in an extensive lattice study of the continuum and infinite volume limits of $\operatorname{Sp}\left(N_{c}\right)$ gauge theories for $N_{c}=2,4,6,8$. We also extrapolate the combined results toward the large- $N_{c}$ limit. We compute the ratio of scalar and tensor masses, and observe evidence that this ratio is independent of $N_{c}$. Other lattice studies of Yang-Mills theories at the same space-time dimension provide a compatible ratio. We further compare these results to various analytical ones and discuss them in view of symmetry-based arguments related to the breaking of scale invariance in the underlying dynamics, showing that a constant ratio might emerge in a scenario in which the $0^{++}$glueball is interpreted as a dilaton state.
\end{abstract}

DOI: 10.1103/PhysRevD.102.011501

\section{INTRODUCTION}

In $D=3+1$ space-time dimensions, Yang-Mills (YM) theories are classically scale-invariant. At high energies the theory is perturbative, and governed by a trivial fixed pointthis is the essence of asymptotic freedom. Scale symmetry is anomalous though, broken by quantum effects that make the theory flow away from its trivial fixed point, and introduce an intrinsic scale $\Lambda$, via dimensional transmutation.

At high energy, the massless gluons, carrying color charges, are the natural choice of degrees of freedom to describe small perturbations around the trivial fixed point. Yang-Mills theories are believed to confine at low energies $\mathcal{O}(\Lambda)$. Low-energy excitations are color singlets, called glueballs, and their spectrum is gapped. The phenomena

\footnotetext{
Corresponding author. dkhong@pusan.ac.kr

Published by the American Physical Society under the terms of the Creative Commons Attribution 4.0 International license. Further distribution of this work must maintain attribution to the author(s) and the published article's title, journal citation, and DOI. Funded by SCOAP .
}

associated with the transition to the confined phase are intrinsically nonperturbative and difficult to study.

In Ref. [1], some of us started an extensive study of $S p\left(N_{c}\right)$ gauge theories, which includes calculating the masses of the glueballs in the YM theory. The spectrum of $S p(4)$ glueballs was one of the most robust results of that exploratory and agenda setting paper. We update the measurements for the $S p(4)$ group, by doubling the size of the combined statistical ensemble, and then proceed to the next step of this program, by performing detailed studies of the YM theory (with no matter content) with gauge groups $S p(2), S p(6)$, and $S p(8)$ (see also preliminary results in Ref. [2]). We report here our results for the lightest scalar and tensor glueballs.

Understanding the glueball spectrum is tantamount to solving the YM theory, and uncovering the mechanism of confinement. Reference [3] suggested that the quantity

$$
R \equiv \frac{m_{2^{++}}}{m_{0^{++}}}
$$

defined as the ratio of masses of the glueballs with quantum number $J^{P C}=2^{++}$and $J^{P C}=0^{++}$, captures some universal, intrinsic properties of YM theories, in the sense that 
it depends only on the dimensionality of the space-time and of the operators of the field theory. We devote this paper to these specific observables. A comprehensive report on the physics of $S p\left(N_{c}\right)$ YM theories, which details the results for excited states and for extended objects, is in preparation [4].

\section{GLUEBALL MASSES: NEW LATTICE RESULTS}

We report at the top of Table I our new lattice measurements of glueball masses in $D=3+1$ dimensions for $S p\left(N_{c}\right)$ YM theories. The algorithm employed in our lattice calculations adopts the Wilson action, and the local updates are based upon a combination of heat bath and over relaxation, by supplementing the Cabibbo-Marinari update with a simple resymplectization procedure, as described in Ref. [1].

We restrict attention to the ratio $m_{G} / \sqrt{\sigma}$ between glueball masses $m_{G}$ and the square root of the string tension $\sigma$. The notation $G=E^{++}, A_{1}^{++}, T_{2}^{++}$, refers explicitly to the representations of the octahedral group, which describes the symmetry of the discretized space-time, and to $P$ and $C$ quantum numbers, as in Ref. [8]. In the measurements, we combine the smearing and blocking of Ref. [5] with the extended basis of operators in the variational approach of Ref. [9].

The errors are due to statistical uncertainties. We perform continuum-limit extrapolations with a conventional linear fit to the dependence on $a^{2}$, where $a$ is the lattice spacing. We also report a simple large- $N_{c}$ extrapolation, in which we include corrections $\mathcal{O}\left(1 / N_{c}\right)$ to $m_{G} / \sqrt{\sigma}$, since the leading corrections occurs at $1 / N_{c}$ [10]. We find that the uncertainty in the string tension $\sigma$ is much smaller than in the masses $m_{G}$. Other technical details, including comments on the systematics and on finite size effects, will appear in Ref. [4].

We identify $m_{A_{1}^{++}}=m_{0^{++}}$. As $m_{E^{++}}$and $m_{T_{2}^{++}}$are compatible with each other, and they both relate to the symmetric tensors in the continuum theory [9], we compute $m_{2^{++}}$as the weighted average of the two. Finally, the error on the ratio $R$ is obtained by simple propagation. The error is overestimated, as we ignore correlations, in particular

TABLE I. Lattice measurements of the masses of the glueballs, as described in the main text. In bold face are the calculations performed for this letter, while the other numerical values are lifted from the literature, as indicated. In the case of $S p(4)$, new measurements have been combined with those from Ref. [1], doubling the combined statistics.

\begin{tabular}{|c|c|c|c|c|c|c|c|}
\hline$D$ & Group & Reference & $\frac{m_{0^{++}}}{\sqrt{\sigma}}=\frac{m_{A_{1}^{++}}}{\sqrt{\sigma}}$ & $\frac{m_{E^{++}}}{\sqrt{\sigma}}$ & $\frac{m_{T_{2}^{++}}}{\sqrt{\sigma}}$ & $\frac{m_{2^{++}}}{\sqrt{\sigma}}$ & $R$ \\
\hline $3+1$ & $S p(2)$ & [4] & $3.841(84)$ & $5.33(18)$ & $5.29(20)$ & $5.31(13)$ & $1.383(46)$ \\
\hline $3+1$ & $S p(4)$ & {$[1,4]$} & $3.729(89)$ & $5.14(16)$ & $5.03(18)$ & $5.09(12)$ & $1.366(45)$ \\
\hline $3+1$ & $S p(6)$ & {$[4]$} & $3.430(75)$ & $5.03(13)$ & $5.09(16)$ & $5.05(10)$ & $1.473(43)$ \\
\hline $3+1$ & $S p(8)$ & [4] & $3.308(98)$ & $4.62(29)$ & $4.73(23)$ & $4.69(18)$ & $1.417(69)$ \\
\hline $3+1$ & $S p(\infty)$ & [4] & $3.241(88)$ & $4.79(19)$ & $4.80(20)$ & $4.80(14)$ & $1.480(58)$ \\
\hline $3+1$ & $S U(2)$ & Table 14 [5] & $3.78(7)$ & $\ldots$ & $\ldots$ & $5.45(11)$ & $1.442(39)$ \\
\hline $3+1$ & $S U(3)$ & Table 14 [5] & $3.55(7)$ & $\cdots$ & $\cdots$ & $4.78(9)$ & $1.346(37)$ \\
\hline $3+1$ & $S U(4)$ & Table 14 [5] & $3.36(6)$ & $\ldots$ & $\ldots$ & $4.88(11)$ & $1.452(42)$ \\
\hline $3+1$ & $S U(6)$ & Table 14 [5] & $3.25(9)$ & $\cdots$ & $\ldots$ & $4.73(15)$ & $1.455(61)$ \\
\hline $3+1$ & $S U(8)$ & Table $14[5]$ & $3.55(12)$ & $\cdots$ & $\ldots$ & $4.73(22)$ & $1.332(77)$ \\
\hline $3+1$ & $S U(\infty)$ & Table 14 [5] & $3.307(53)$ & $\cdots$ & $\cdots$ & $4.80(14)$ & $1.451(48)$ \\
\hline $2+1$ & $S O(3)$ & Table 28 [6] & $3.132(34)$ & $\cdots$ & $\ldots$ & $5.13(9)$ & $1.638(34)$ \\
\hline $2+1$ & $S O(4)$ & Table 28 [6] & $3.343(23)$ & $\cdots$ & $\ldots$ & $5.711(81)$ & $1.708(27)$ \\
\hline $2+1$ & $S O(5)$ & Table 28 [6] & $3.545(17)$ & $\cdots$ & $\cdots$ & $6.008(46)$ & $1.695(15)$ \\
\hline $2+1$ & $S O(6)$ & Table 28 [6] & $3.656(13)$ & $\cdots$ & $\cdots$ & $6.190(38)$ & $1.693(12)$ \\
\hline $2+1$ & $S O(7)$ & Table 29 [6] & $3.737(10)$ & $\cdots$ & $\cdots$ & $6.297(54)$ & $1.685(15)$ \\
\hline $2+1$ & $S O(8)$ & Table 29 [6] & $3.788(14)$ & $\cdots$ & $\cdots$ & $6.498(36)$ & $1.715(11)$ \\
\hline $2+1$ & $S O(12)$ & Table 29 [6] & $3.878(24)$ & $\ldots$ & $\cdots$ & $6.636(64)$ & $1.711(20)$ \\
\hline $2+1$ & $S O(16)$ & Table $29[6]$ & $3.973(15)$ & $\cdots$ & $\cdots$ & $6.714(40)$ & $1.690(12)$ \\
\hline $2+1$ & $S O(\infty)$ & Table 31 [6] & $4.150(33)$ & $\cdots$ & $\cdots$ & $6.987(88)$ & $1.684(25)$ \\
\hline $2+1$ & $S O(\infty)$ & Table 31 [6] & $4.179(16)$ & $\cdots$ & $\cdots$ & $7.129(43)$ & $1.706(12)$ \\
\hline $2+1$ & $S U(2)$ & Table B3 [7] & $4.7369(55)$ & $\ldots$ & $\ldots$ & $7.762(10)$ & $1.6386(28)$ \\
\hline $2+1$ & $S U(3)$ & Table B4 [7] & $4.3683(73)$ & $\cdots$ & $\cdots$ & 7.241(17) & $1.6576(48)$ \\
\hline $2+1$ & $S U(4)$ & Table B5 [7] & $4.242(9)$ & $\ldots$ & $\ldots$ & 7.091(17) & $1.6616(54)$ \\
\hline $2+1$ & $S U(6)$ & Table B6 [7] & $4.164(8)$ & $\cdots$ & $\ldots$ & $6.983(19)$ & $1.6770(56)$ \\
\hline $2+1$ & $S U(8)$ & Table B7 [7] & $4.144(10)$ & $\cdots$ & $\ldots$ & $6.952(18)$ & $1.6776(59)$ \\
\hline $2+1$ & $S U(12)$ & Table B8 [7] & $4.140(9)$ & $\cdots$ & $\cdots$ & $6.938(18)$ & $1.6759(57)$ \\
\hline $2+1$ & $S U(16)$ & Table B9 [7] & $4.129(11)$ & $\cdots$ & $\cdots$ & $6.937(30)$ & $1.6801(85)$ \\
\hline $2+1$ & $S U(\infty)$ & Tables B10,B11 [7] & $4.116(6)$ & $\cdots$ & $\cdots$ & $6.914(13)$ & $1.6798(40)$ \\
\hline
\end{tabular}




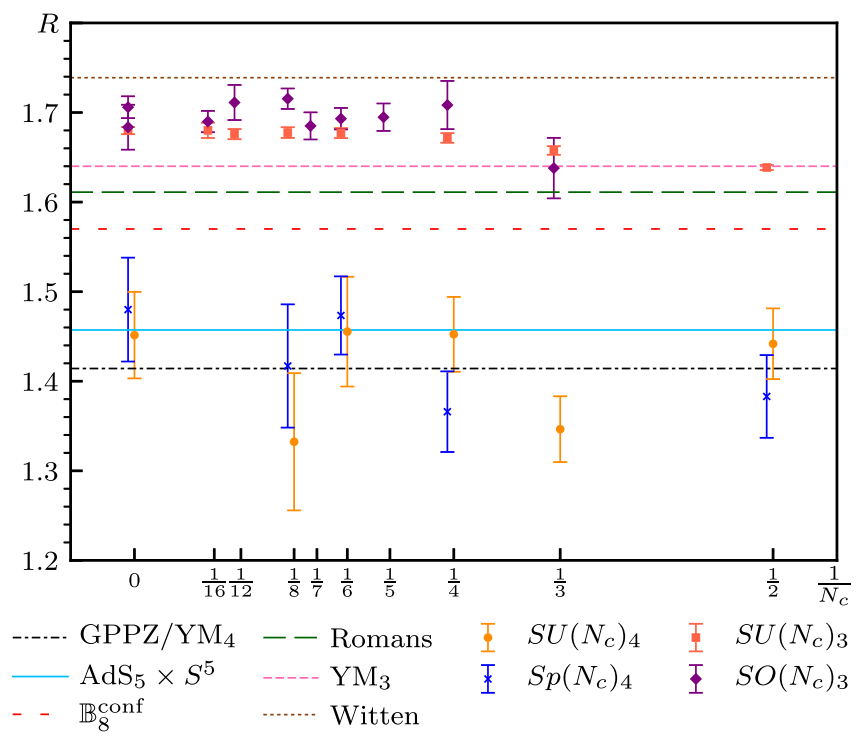

FIG. 1. Numerical and analytical results for the ratio $R$ defined in Eq. (1). Different shaped markers denote the lattice measurements with continuum extrapolations in $D=3+1$ dimensions for $\operatorname{Sp}\left(N_{c}\right)$ and for $S U\left(N_{c}\right)$ [5], as well as in $D=2+1$ dimensions for $S O\left(N_{c}\right)$ [6] and $S U\left(N_{c}\right)$ [7]. Extrapolations to the $N_{c} \rightarrow \infty$ limit are also included. Differently rendered lines at $R=\sqrt{2}, 1.46,1.57,1.61,1.74$, are the holographic calculations in the GPPZ model [11], the circle reduction of $\mathrm{AdS}_{5} \times S^{5}$ $[12,13]$, the holographic model $\mathbb{B}_{8}^{\text {conf }}$ in Ref. [14], the Witten model $[12,15]$, and the circle reduction of Romans supergravity [15,16], respectively. With $R=\sqrt{2}, 1.64$ we report the field theoretical results from Refs. [17,18], for YM theories in $D=$ $3+1$ and $D=2+1$ dimensions, respectively. More details can be found in the main text.

because of the common dependence on $\sigma$, but we expect such effects to be small, and not to affect our discussion.

Figure 1 shows that the ratio $R$ for the sequence of $S p\left(N_{c}\right)$ YM theories is compatible with a constant. This confirms that $O\left(1 / N_{c}\right)$ effects, if present, are smaller than the current uncertainties, the magnitude of which varies between $\sim 2 \%$ for $S p(4)$ and $5 \%$ for $S p(8)$.

\section{GLUEBALL MASSES: EARLIER LATTICE RESULTS}

We include in Table I and Fig. 1 our measurements (denoted $S p\left(N_{c}\right)_{4}$ ), together with lattice results by other collaborations, for various classes of YM theories.

The spectrum of YM glueballs in $D=3+1$ dimensions with $S U\left(N_{c}\right)$ group (denoted $S U\left(N_{c}\right)_{4}$ ) was studied in Refs. [5,9]. In the former, the authors use a single value of the lattice parameters for each value of $N_{c}$, without studying the approach to the continuum limit. Conversely, Ref. [5] reports continuum limits for the glueball masses expressed in units of the string tension $\sigma$, but the variational method uses a smaller basis of operators of the octahedral group in respect to our work, and the $T_{2}$ channel is not measured.
As long as we restrict attention to the lightest states in the spectrum (the $0^{++}$and $2^{++}$ground states), at the same lattice spacing the results of the two approaches are in good agreement, and hence we compare the $\operatorname{Sp}\left(N_{c}\right)$ sequence of measurements, as well as their extrapolation to large $N_{c}$, to those of Ref. [5]. As visible in Fig. 1, the agreement in the ratio $R$ across the gauge groups is excellent.

We also summarize the lattice measurements for $S O\left(N_{c}\right)$ in $D=2+1$ dimensions $\left(S O\left(N_{c}\right)_{3}\right)$, taken from Tables 28, 29 and 31 of Ref. [6] (see also Fig. 26 therein). We include only continuum limit results, and two different types of large- $N_{c}$ extrapolations. Finally, we collect results for $S U\left(N_{c}\right)$ theories in $D=2+1$ dimensions $\left(S U\left(N_{c}\right)_{3}\right)$ from Tables B3-B11 of Ref. [7]. The extrapolation to $S U(\infty)$ has been performed by including $1 / N_{c}^{2}$ as well as $1 / N_{c}^{4}$ corrections.

Lattice results on $R$ show the emergence of a regular pattern, that depends only on the dimensionality $D$ of the system. The group sequence $\left[S U\left(N_{c}\right), S p\left(N_{c}\right)\right.$ or $\left.S O\left(N_{c}\right)\right]$ and the number of colors $N_{c}$ do not appear to affect $R$, within current uncertainties-with some deviation from this pattern in $D=2+1$ dimensions for $S U(3), S O(3)$ and $S U(2)$. We have at our disposal preliminary results for excited states and states with different quantum numbers in $S p\left(N_{c}\right)$ theories (to appear in Ref. [4]), and we did not find significant evidence of similar regular patterns, reinforcing the notion that the lightest $0^{++}$and $2^{++}$glueballs play a special role in YM theories.

\section{GLUEBALL MASSES: A BRIEF SURVEY OF ANALYTICAL RESULTS}

In Fig. 1, we compare the result of lattice measurements of the ratio $R$ to two classes of semianalytical calculations, performed either via gauge-gravity dualities arising in the context of supergravity, or via alternative field-theory methods. In all these models, the ratio $R$ is known only in the strict large- $N_{c}$ limit, as $1 / N_{c}$ corrections are ignored.

The GPPZ model was proposed in Ref. [19] (see also Refs. [20-22]) as a simple, classical supergravity dual of mass-deformed, large- $N_{c}, \mathcal{N}=4$ Super-Yang-Mills. The geometry is singular and asymptotically approaches $\mathrm{AdS}_{5}$. The spectrum of fluctuations yields $R=\sqrt{2}$ [11] (see also Refs. [23-25]). This result happens to be in exact agreement with that of the large- $N_{c}$ field-theory study in Ref. [17] (see Table 1 therein), which in Fig. 1 we denote as $\mathrm{YM}_{4}$. A closely related model is studied in Ref. [12], that reports a holographic calculation based upon the circle reduction of the system yielding the $\operatorname{AdS}_{5} \times S^{5}$ background (see also Ref. [13]). The result in this case is $R=1.46$. The close proximity between the results of these two holographic calculations (both of which use geometries that are asymptotically $\mathrm{AdS}_{5}$ ), Bochicchio's field-theoretical approach [17,26], and lattice calculations in $\operatorname{Sp}\left(N_{c}\right)$ and $\mathrm{SU}\left(N_{c}\right)$ is remarkable. 
Witten's holographic model of confinement [27] is based upon $S^{1} \times S^{1} \times S^{4}$ reduction of eleven-dimensional supergravity [28-31]. In the asymptotically $\mathrm{AdS}_{7}$ background geometry, one $S^{1}$ shrinks to zero size. The static quarkantiquark potential is computed holographically [32,33], and yields linear confinement. Adaptations to model quenched QCD were proposed in Refs. [34,35]. The spectrum of glueballs yields $R=1.74$ [12] (see also Ref. [15]). An alternative model, based on circle reduction of Romans supergravity [36], has geometry that is asymptotically $\mathrm{AdS}_{6}$, and again the circle shrinks. In this case, $R=1.61$ [16] (see also Refs. [15,37,38]). For both celebrated models, Fig. 1 shows that $R$ is not compatible with the lattice results, with current uncertainties.

The literature on the holographic dual of threedimensional confining theories is more limited. In Ref. [14] the model dubbed $\mathbb{B}_{8}^{\text {conf }}$ is the gravity dual of a nontrivial, asymptotically free theory in $2+1$ dimensions [39-42], and yields $R \simeq 1.57$. A completely different fieldtheory approach to YM theories in $2+1$ dimensions is used to compute glueball masses in Refs. [18,43] (we denote it as $\mathrm{YM}_{3}$ in Fig. 1). From the latter of the two, we read that $R \simeq 1.64$. This result is valid only in the strict $N_{c} \rightarrow+\infty$ limit, although the analysis in Ref. [18] could potentially be extended to finite $N_{c}$. Both these approaches $\left(\mathbb{B}_{8}^{\text {conf }}\right.$ and $\mathrm{YM}_{3}$ in Fig. 1) slightly underestimate $R$ in respect to the lattice results for $S U\left(N_{c}\right)$ and $S O\left(N_{c}\right)$.

\section{DISCUSSIONS AND UNIVERSAL RATIO}

If the ratio between the masses of the lightest spin-2 and spin-0 glueballs is universal for (pure) YM theories, there should be underlying principles that hold for all of them. We argue (see also Ref. [44]) that scale symmetry and perturbative unitarity are such principles.

When the YM theory undergoes the phase transition to the confining phase, the vacuum energy density $\mathcal{E}_{\text {vac }}$ is lowered, breaking scale invariance spontaneously, to yield

$$
\mathcal{E}_{\mathrm{vac}} \equiv \frac{1}{4}\left\langle T_{\mu}^{\mu}\right\rangle<0,
$$

with $T_{\mu \nu}$ the energy-momentum tensor.

As the vacuum is not invariant under scale transformations, the dilatation current $D_{\mu}=x^{\nu} T_{\mu \nu}$ creates a state, called a dilaton, out of the vacuum, which we write as

$$
\left\langle 0\left|D_{\mu}(x)\right| \sigma(p)\right\rangle \equiv i f_{D} p_{\mu} e^{-i p \cdot x},
$$

where $f_{D}$ is the dilaton decay constant. If the two-point function of dilatation currents is dominated by the dilaton pole at low energy, for $p \rightarrow 0$ we expect:

$$
\int_{x} e^{i p \cdot x}\left\langle 0\left|\mathrm{~T}\left[T_{\mu}^{\mu}(x) T_{\nu}^{\nu}(0)\right]\right| 0\right\rangle \approx f_{D}^{2} m_{D}^{2}=-16 \mathcal{E}_{\mathrm{vac}}
$$

with $m_{D}$ being the dilaton mass. Under this assumption, we identify the ground-state glueball with the dilaton, because it is the lightest particle and both of them have the same quantum numbers as the vacuum. How good this approximation is can only be assessed a posteriori.

The Lagrangian density of the dilaton low-energy effective field theory (EFT) is the subject of a vast literature. The potential must break scale invariance explicitly, and contain nonmarginal operators. Departures from marginality might be encoded in a logarithmic field-dependent potential, as advocated in Refs. [45,46]. (More general, power-law potentials have also been considered [47-54]). We dispense with such level of detail in the context of this discussion. It is natural to assume that the intrinsic, dynamically generated scale $\Lambda$ sets $\mathcal{E}_{\text {vac }} \sim \Lambda^{4}$ and $f_{D} \sim \Lambda$. Therefore, from Eq. (4) and taking $16 \mathcal{E}_{\mathrm{vac}}=-\beta f_{D}^{4}$, we may write

$$
f_{D}^{2} m_{D}^{2}=\beta f_{D}^{4}
$$

The numerical constant $\beta$ is an intrinsic constant of the YM theory, and depends on the gauge group. It measures the size of explicit breaking of scale symmetry, sets the strength of the self-interaction of the dilaton, and is the expansion parameter of the EFT. The parameter $\beta$ is not guaranteed to be small. Lattice calculations find that the spin- 2 glueball is the lowest excited state, and has mass of the same order of magnitude as that of the ground-state glueball.

The dilaton EFT yields the amplitude $\mathcal{M}_{\sigma}$, for the scattering process $\sigma\left(p_{1}\right)+\sigma\left(p_{2}\right) \rightarrow \sigma\left(p_{3}\right)+\sigma\left(p_{4}\right)$ between dilaton particles. For center-of-mass energies $E \gg m_{D}$, we borrow Eq. (3.3) from Ref. [55] (see also Ref. [56]) and write

$$
\mathcal{M}_{\sigma} \sim-\frac{1}{\alpha^{4} f_{D}^{4}}\left(s^{2}+t^{2}+u^{2}\right)+\mathcal{O}\left(\frac{m_{D}^{2}}{f_{D}^{2}}\right),
$$

in terms of the Mandelstam variables $s=\left(p_{1}+p_{2}\right)^{2}$, $t=\left(p_{3}-p_{1}\right)^{2}$, and $u=\left(p_{4}-p_{1}\right)^{2}$. Here $\alpha$ is a dimensionless constant characterizing the theory. The scattering amplitude violates perturbative unitarity at $E \sim \alpha f_{D}$, To achieve partial unitarity restoration, and raise this bound, we introduce the spin-2 glueball in the EFT. We assume that the spin-2 glueball couples to the energy-momentum tensor of the dilaton $T_{D}^{\mu \nu}$.

The Lagrangian density of the massive spin-2 glueball $h_{\mu \nu}$ can be derived by identifying it with the expansion of the spacetime metric around the flat spacetime as in $g_{\mu \nu}=\eta_{\mu \nu}+2 \kappa h_{\mu \nu}$, to obtain

$$
\mathcal{L}_{G}=\mathcal{L}_{G}^{\mathrm{kin}}-\kappa h_{\mu \nu} T_{D}^{\mu \nu}+\cdots,
$$

where the first term is the so-called Fierz-Pauli kinetic-term for the massive spin-2 fields, $\kappa$ is the (universal) coupling 
of the spin-2 glueballs and the ellipsis denotes the higher order terms. Again, the assumptions underneath this identification can be assessed a posteriori.

The propagator of the massive spin- 2 field of mass $m_{T}$ is then given by [57]

$$
\int_{x} e^{i p \cdot x}\left\langle 0\left|T\left\{h_{\mu \nu}(x) h_{\alpha \beta}(0)\right\}\right| 0\right\rangle=\frac{i P_{\mu \nu \alpha \beta}}{p^{2}-m_{T}^{2}+i \epsilon},
$$

where $2 P_{\mu \nu \alpha \beta}=\tilde{\eta}_{\mu \alpha} \tilde{\eta}_{\nu \beta}+\tilde{\eta}_{\mu \beta} \tilde{\eta}_{\nu \alpha}-\frac{2}{3} \tilde{\eta}_{\mu \nu} \tilde{\eta}_{\alpha \beta} \quad$ with $\quad \tilde{\eta}_{\mu \nu}=$ $\eta_{\mu \nu}-p_{\mu} p_{\nu} / m_{T}^{2}$. The contribution of the diagrams with internal exchange of the spin-2 particles changes the structure of the amplitude, and partially restores perturbative unitarity to hold at the scale $E \sim\left(\kappa f_{D}\right)^{-1} \cdot m_{T}$ and slightly above, where $\kappa f_{D}$ measures the strength of the spin-2 coupling to the dilaton, compared to the dilaton self-coupling. For this to happen, one must require that $\alpha f_{D} \sim\left(\kappa f_{D}\right)^{-1} \cdot m_{T}$, or $m_{T}^{2} \equiv g f_{D}^{2} \sim \alpha \kappa^{2} f_{D}^{4}$.

The dimensionless constant $g \sim \alpha \kappa^{2} f_{D}^{2}$ depends on the microscopic details of the theory, as $\beta$. Combining this with Eq. (5), we write the mass ratio of the spin-2 glueball and the ground-state glueball as

$$
R^{2} \equiv \frac{m_{T}^{2}}{m_{D}^{2}}=\frac{g}{\beta}
$$

In the mass ratio between the lightest spin- 2 and spin- 0 glueball the dependence on microscopic details should decouple as suggested by the lattice data. As the EFT captures the long-distance dynamics based on symmetry (and perturbative unitarity) considerations, that are common to all YM theories, it should describe all lowenergy (pure) YM theories.

The lattice data we summarized suggests the ratio $R$ in $D=2+1$ is also universal. It has been noted elsewhere that the similarities between the physics of confinement in $D=2+1$ and in $D=3+1$ dimensions turn out to be much deeper than naively expected (see e.g., Ref. [58]). On this basis, we argue that also in $D=2+1$ dimensions the constant ratio is controlled by spontaneous as well as explicit breaking of scale invariance through confinement, which, by generating a mass gap, changes the would-be power law behavior of gluon correlators, at distances much larger than the intrinsic length scale set by the dimensional gauge coupling.

\section{OUTLOOK}

Our lattice measurements of the masses of the lightest scalar and tensor glueballs for $\operatorname{Sp}\left(N_{c}\right)$ gauge theories in $D=3+1$ dimensions show no discernible dependence on $N_{c}$ in the ratio $R$ defined by Eq. (1). We compared this finding with lattice measurements taken from the literature, and compiled a (non exhaustive) list of other calculations, that use holography or alternative field theory methods.
We found supporting empirical evidence that the ratio $R$ might be a universal quantity in YM theories, in the sense that it appears to depend only on the dimensionality of the system, not its microscopic details.

This intriguing feature might be connected with the special role that the lightest scalar glueball and the lightest tensor glueball play in respect to scale invariance. As we argued in Sec. V, it might be explained under the approximation that these two particles can be identified with those sourced by the dilatation operator and by the energymomentum tensor. This approximation relies on two separate assumptions: that the explicit breaking of scale invariance is small compared to its spontaneous breaking, and that single particle exchange saturates the 2-point correlation functions build with the dilatation operator and the energy-momentum tensor.

Our arguments highlight the distinguishing features of the two particles that are the main topic of this paper. More theoretical work would be useful, to better understand the role of these two particles, and whether the empirical evidence we uncovered points to an exact relation, or, if otherwise, to estimate the size of deviations. It would also be very useful to have lattice data on Yang-Mills theories with other gauge groups, and we hope such calculations will be performed in the future.

\section{ACKNOWLEDGMENTS}

We thank D. Elander for discussion about Ref. [14]. The work of E. B. has been funded by the Supercomputing Wales project, which is part-funded by the European Regional Development Fund (ERDF) via Welsh Government. J.H. is supported by the STFC Consolidated Grant No. ST/P00055X/1, by the College of Science, Swansea University, and by the Grant No. STFC-DTG ST/R505158/1. The work of D. K. H. was supported by Basic Science Research Program through the National Research Foundation of Korea (NRF) funded by the Ministry of Education (Grant No. NRF-2017R1D1A1B06033701) and he is grateful to CERN-TH for the hospitality, where the part of this work is done. The work of J. W. L. is supported in part by the National Research Foundation of Korea grant funded by the Korea government (MSIT) (No. NRF2018R1C1B3001379) and in part by Korea Research Fellowship programme funded by the Ministry of Science, ICT and Future Planning through the National Research Foundation of Korea (Grant No. 2016H1D3A1909283). The work of C.J.D.L. is supported by the Taiwanese MoST Grant No. 105-2628-M-009-003-MY4. The work of B.L. and M.P. has been supported in part by the STFC Consolidated Grants No. ST/L000369/1 and No. ST/P00055X/1. B. L. and M. P. received funding from the European Research Council (ERC) under the European Union's Horizon 2020 research and innovation programme under Grant Agreement No. 813942. The work of B. L. is further supported in part by the Royal Society Wolfson 
Research Merit Award No. WM170010. D. V. is supported by the INFN HPC-HTC project. Numerical simulations have been performed on the Swansea SUNBIRD system, on the local HPC clusters in Pusan National University (PNU) and in National Chiao-Tung University (NCTU), and on the Cambridge Service for Data Driven Discovery (CSD3). The Swansea SUNBIRD system is part of the Supercomputing Wales project, which is part-funded by the European
Regional Development Fund (ERDF) via Welsh Government. CSD3 is operated in part by the University of Cambridge Research Computing on behalf of the STFC DiRAC HPC Facility [59]. The DiRAC component of CSD3 was funded by BEIS capital funding via STFC capital Grants No. ST/P002307/1 and No. ST/R002452/1 and STFC operations Grant No. ST/R00689X/1. DiRAC is part of the National e-Infrastructure.
[1] E. Bennett, D. K. Hong, J. W. Lee, C.-J. D. Lin, B. Lucini, M. Piai, and D. Vadacchino, J. High Energy Phys. 03 (2018) 185.

[2] J. Holligan, E. Bennett, D. K. Hong, J. W. Lee, C.-J. D. Lin, B. Lucini, M. Piai, and D. Vadacchino, arXiv:1912.09788.

[3] A. Athenodorou, E. Bennett, G. Bergner, D. Elander, C.-J. D. Lin, B. Lucini, and M. Piai, J. High Energy Phys. 06 (2016) 114.

[4] J. Holligan, E. Bennett, D. K. Hong, J. W. Lee, C.-J. D. Lin, B. Lucini, M. Piai, and D. Vadacchino (to be published).

[5] B. Lucini, M. Teper, and U. Wenger, J. High Energy Phys. 06 (2004) 012.

[6] R. Lau and M. Teper, J. High Energy Phys. 10 (2017) 022.

[7] A. Athenodorou and M. Teper, J. High Energy Phys. 02 (2017) 015.

[8] B. Berg and A. Billoire, Nucl. Phys. B221, 109 (1983).

[9] B. Lucini, A. Rago, and E. Rinaldi, J. High Energy Phys. 08 (2010) 119.

[10] C. Lovelace, Nucl. Phys. B201, 333 (1982).

[11] W. Mueck and M. Prisco, J. High Energy Phys. 04 (2004) 037.

[12] R. C. Brower, S. D. Mathur, and C. I. Tan, Nucl. Phys. B587, 249 (2000).

[13] D. Elander, M. Piai, and J. Roughley, J. High Energy Phys. 06 (2020) 177.

[14] D. Elander, A. F. Faedo, D. Mateos, D. Pravos, and J. G. Subils, J. High Energy Phys. 05 (2019) 175.

[15] D. Elander, A. F. Faedo, C. Hoyos, D. Mateos, and M. Piai, J. High Energy Phys. 05 (2014) 003.

[16] C. K. Wen and H. X. Yang, Mod. Phys. Lett. A 20, 997 (2005).

[17] M. Bochicchio, arXiv:1308.2925.

[18] R. G. Leigh, D. Minic, and A. Yelnikov, Phys. Rev. D 76, 065018 (2007).

[19] L. Girardello, M. Petrini, M. Porrati, and A. Zaffaroni, Nucl. Phys. B569, 451 (2000).

[20] L. Girardello, M. Petrini, M. Porrati, and A. Zaffaroni, J. High Energy Phys. 12 (1998) 022.

[21] J. Distler and F. Zamora, Adv. Theor. Math. Phys. 2, 1405 (1999).

[22] K. Pilch and N. P. Warner, Adv. Theor. Math. Phys. 4, 627 (2002).

[23] R. Apreda, D. E. Crooks, N. J. Evans, and M. Petrini, J. High Energy Phys. 05 (2004) 065.

[24] D. Elander and M. Piai, Nucl. Phys. B864, 241 (2012).
[25] D. Elander and M. Piai, Nucl. Phys. B867, 779 (2013).

[26] M. Bochicchio, AIP Conf. Proc. 1735, 030004 (2016).

[27] E. Witten, Adv. Theor. Math. Phys. 2, 505 (1998).

[28] H. Nastase, D. Vaman, and P. van Nieuwenhuizen, Phys. Lett. B 469, 96 (1999).

[29] M. Pernici, K. Pilch, and P. van Nieuwenhuizen, Phys. Lett. 143B, 103 (1984).

[30] M. Pernici, K. Pilch, P. van Nieuwenhuizen, and N. P. Warner, Nucl. Phys. B249, 381 (1985).

[31] H. Lu and C. N. Pope, Phys. Lett. B 467, 67 (1999).

[32] J. M. Maldacena, Phys. Rev. Lett. 80, 4859 (1998).

[33] S. J. Rey and J. T. Yee, Eur. Phys. J. C 22, 379 (2001).

[34] T. Sakai and S. Sugimoto, Prog. Theor. Phys. 113, 843 (2005).

[35] T. Sakai and S. Sugimoto, Prog. Theor. Phys. 114, 1083 (2005).

[36] L. J. Romans, Nucl. Phys. B269, 691 (1986).

[37] S. Kuperstein and J. Sonnenschein, J. High Energy Phys. 11 (2004) 026.

[38] D. Elander, M. Piai, and J. Roughley, J. High Energy Phys. 02 (2019) 101.

[39] G. W. Gibbons, D. N. Page, and C. N. Pope, Commun. Math. Phys. 127, 529 (1990).

[40] A. Hashimoto, S. Hirano, and P. Ouyang, J. High Energy Phys. 06 (2011) 101.

[41] M. Cvetic, G. W. Gibbons, H. Lu, and C. N. Pope, J. Geom. Phys. 49, 350 (2004).

[42] A. F. Faedo, D. Mateos, D. Pravos, and J. G. Subils, J. High Energy Phys. 06 (2017) 153.

[43] R. G. Leigh, D. Minic, and A. Yelnikov, Phys. Rev. Lett. 96, 222001 (2006).

[44] D. K. Hong, J. W. Lee, B. Lucini, M. Piai, and D. Vadacchino, Phys. Lett. B 775, 89 (2017).

[45] J. Schechter, Phys. Rev. D 21, 3393 (1980).

[46] A. A. Migdal and M. A. Shifman, Phys. Lett. 114B, 445 (1982).

[47] R. Rattazzi and A. Zaffaroni, J. High Energy Phys. 04 (2001) 021.

[48] Z. Chacko and R. K. Mishra, Phys. Rev. D 87, 115006 (2013).

[49] T. Appelquist, J. Ingoldby, and M. Piai, J. High Energy Phys. 07 (2017) 035.

[50] T. Appelquist, J. Ingoldby, and M. Piai, J. High Energy Phys. 03 (2018) 039. 
[51] O. Catà, R. J. Crewther, and L. C. Tunstall, Phys. Rev. D 100, 095007 (2019).

[52] O. Catà and C. Mueller, Nucl. Phys. B952, 114938 (2020).

[53] T. Appelquist, J. Ingoldby, and M. Piai, Phys. Rev. D 101, 075025 (2020).

[54] Z. Fodor, K. Holland, J. Kuti, and C. H. Wong, arXiv: 2002.05163.
[55] Z. Komargodski and A. Schwimmer, J. High Energy Phys. 12 (2011) 099.

[56] D. K. Hong et al. (to be published).

[57] M. Fierz and W. Pauli, Proc. R. Soc. A 173, 211 (1939).

[58] M. J. Teper, Phys. Rev. D 59, 014512 (1999).

[59] www.dirac.ac.uk. 